\title{
Labyrinthe
}

$5 \mid 2000$

Numéro 5

\section{Intervention des femmes et redéfinition de l'espace public}

Le cas de l'Association des femmes siciliennes pour la lutte contre la mafia

Karine Delavie-Blanchon

\section{OpenEdition}

Journals

Édition électronique

URL : http://journals.openedition.org/labyrinthe/260

DOI : $10.4000 /$ labyrinthe. 260

ISSN : 1950-6031

Éditeur

Hermann

Édition imprimée

Date de publication : 15 janvier 2000

Pagination : 43-59

Référence électronique

Karine Delavie-Blanchon, «Intervention des femmes et redéfinition de l'espace public », Labyrinthe [En ligne], 5 | 2000, mis en ligne le 16 février 2005, consulté le 19 avril 2019. URL : http://

journals.openedition.org/labyrinthe/260; DOI : 10.4000/labyrinthe.260

Ce document a été généré automatiquement le 19 avril 2019

Propriété intellectuelle 


\title{
Intervention des femmes et redéfinition de l'espace public
}

\author{
Le cas de l'Association des femmes siciliennes pour la lutte contre la \\ mafia
}

\author{
Karine Delavie-Blanchon
}

Avant de combattre la mafia, tu dois faire un autoexamen de conscience, et ensuite, après avoir battu la mafia en toi-même, tu peux combattre la mafia qui est dans le milieu de tes amis ; la mafia,

c'est nous et notre manière fausse de nous comporter ${ }^{1}$. (Rita Atria)

Femmes et mafia ont pendant longtemps été considérées comme deux " mondes » antinomiques ; les femmes étaient à la fois complètement exclues de cette " affaire d'hommes » et de l'espace public sicilien. Pourtant, l'investissement de femmes dans la lutte antimafia est ancien ; dès les fasci siciliens (1892-1894), elles y occupaient une place très importante. Plus tard, après la chute du fascisme qui avait férocement combattu Cosa Nostra - notamment avec l'action du célèbre préfet Mori -, la mafia retrouve son ancienne influence et les syndicalistes et militants socialistes entament une nouvelle phase de lutte. Bon nombre d'entre eux sont assassinés sans que leurs meurtriers soient recherchés. C'est à ce moment que pour la première fois de l'histoire sicilienne, des femmes demandent justice ; Francesca Serio ${ }^{2}$ en 1955 et Serafina Battaglia ${ }^{3}$ en 1960 deviennent des emblèmes de courage. Les années 80 marquent le début du « mouvement de masse » qui trouve son paroxysme au début des années 90 notamment en prenant pour ressource l'émotion collective avec le Comité des draps et l'action des femmes du jeûne en 1992, en réaction aux massacres de Capaci - assassinats de Giovanni Falcone, de sa femme et de trois gardes du corps - et de Via D'Amelio - assassinat de Paolo Borsellino et de son escorte. C'est précisément à partir du milieu des années 90 que de nombreuses associations antimafia se créent. Mais la première d'entre elles, l'Association des femmes siciliennes pour la lutte contre la mafia naît en 1984 et se définit comme exclusivement 
féminine. Pourquoi ? Pour apporter des éléments de réponse, il est important de comprendre les raisons et le contexte de sa création, et d'analyser qui sont ces femmes*.

\section{S'ENGAGER EN TANT QUE FEMME CONTRE LA MAFIA}

Pour saisir les raisons de cette action associative féminine, il semble nécessaire de situer les questions de genre dans le contexte sicilien, mais aussi dans l'organisation mafieuse, parce que si l'on considère qu'il y a une spécificité des femmes en matière de lutte antimafia, peut-être faut-il poser l'hypothèse qu'il y a, parallèlement, une spécificité des femmes à l'intérieur de la mafia.

La mafia à travers la lecture du genre

3 La principale caractéristique de la mafia sicilienne est d'être clairement monosexuelle ; traditionnellement, elle est une " affaire d'hommes ", et la femme y tient une place subordonnée et silencieuse ; par exemple, « la femme de Calderone [...] est une parfaite "femme d'homme d'honneur", affectueuse, discrète, efficace, ne disant jamais un mot de trop et montrant une dévotion sans bornes ${ }^{4}$. » L'homme mafieux est un " mari-patron », un " père-patron ", ou un " frère-patron » qui a fait serment de tout subordonner aux règles de l'Honorable Société. Le système de représentation mafieux a construit une identité féminine dangereuse, fragile et fourbe, c'est pourquoi, indigne de confiance, la femme est exclue de la sphère mafieuse, dans laquelle elle n'a de place qu'en tant que mère - mammasantissima. L'arrêt du 11 février 1983 du Tribunal civil et pénal de Palerme illustre cette subordination en disculpant

quelques femmes de boss indiscutés, impliquées comme prête-noms et intermédiaires dans des affaires se montant à des milliards, au motif de leur insuffisante possibilité d'émancipation dans le milieu mafieux et de leur « naturelle et traditionnelle étrangéité » au monde difficile des affaires.

Dans l'esprit de la mafia, la femme n'est acceptable qu'en tant que « mère de ses enfants ». Ses fonctions principales sont celles de reproductrice et d'éducatrice et sont révélatrices du rôle de la femme dans la transmission des normes mafieuses telles que l' omertà et la vendetta. Michela Buscemi, sœur de deux mafieux assassinés, m'explique :

Les femmes élèvent les enfants dans la culture mafieuse la plus radicale. Si le mari mafieux est tué, c'est la femme qui inculque à ses fils la vendetta. Celui qui a tué le mari doit être tué à son tour. Le mari doit être vengé. La femme pousse les enfants à être encore plus mafieux que le père ${ }^{5}$.

5 La femme est exclue, mais la mère semble tenir un rôle clef. Certes, certaines études récentes tendent à montrer une participation féminine nouvelle et grandissante dans l'organisation ${ }^{6}$. Néanmoins, plus que leur nombre, c'est leur rôle et leur pouvoir réel qui est significatif, et l'on sait que leur participation ne signifie pas pour Cosa Nostra affiliation, mais plus souvent exploitation. Par ailleurs, celles qui y appartiennent n'ont généralement pas eu le choix ; soit elles sont nées dans une famille mafieuse, soit elles y ont été affiliées par mariage. Enfin, s'il est vrai que ces dernières années, les journaux italiens se sont fortement intéressés aux quelques " femmes boss ", peut-on véritablement parler d'émancipation alors qu'elles ne font que suppléer leurs maris emprisonnés, encadrées par de strictes règles sans aucune liberté ? Finalement, la mafia était et reste une organisation monosexuelle. Or l'on sait que culture mafieuse et culture sicilienne sont intrinsèquement liées. Anna Puglisi affirme par exemple : "Je suis convaincue que la monosexualité de l'organisation mafieuse ne lui est pas spécifique mais qu'elle est un reflet du machisme de notre société ${ }^{7}$ ", et Giovanni Falcone abonde en son sens : « [...] La mafia n'est rien d'autre que la distorsion, l'exagération, l'extrémisation aberrante des manières d'être du Sicilien ${ }^{8}$. " Faut-il dès lors considérer que lorsqu'est 
fondée l'Association des femmes siciliennes pour la lutte contre la mafia, les femmes siciliennes étaient également bannies de la sphère publique et renvoyées à la sphère privée ?

Les femmes et la res publica en Sicile

De nombreux stéréotypes affectent les femmes siciliennes parmi lesquels la thèse du matriarcat, qui oppose à une apparente invisibilité dans la sphère publique, un pouvoir absolu au sein de la sphère privée. Il me semble que si la femme a une position centrale dans la famille méridionale ${ }^{9}$, il faut néanmoins nuancer cette position, car elle est alors renvoyée à son rôle de mère. D'ailleurs, si le taux de fécondité italien est aujourd'hui le plus bas d'Europe, il demeure en Sicile relativement élevé - même s'il décroît depuis quelques années : "La Sicile est [...] l'une des dernières régions d'Italie à ne pas être frappée par le problème de la dénatalité (le taux de natalité y est de 2,3 enfants par femme contre 1,3 pour la moyenne italienne). ${ }^{10}$ »

7 Il y a donc une tradition d'enfermement dans la sphère privée. En effet, les femmes sont quasiment absentes de l'espace public depuis longtemps, les deux pôles les plus significatifs étant celui du marché du travail et de la politique. Le taux d'inactivité des femmes est très élevé en Sicile, et elles étaient par ailleurs absentes des affaires publiques jusqu'à une date récente - il suffit de regarder le nombre peu élevé de députées élues pour s'en convaincre.

8 Parallèlement à son éviction de Cosa Nostra, il semble en effet qu'il y ait eu une tradition d'exclusion de la femme de la sphère publique sicilienne, elle-même dominée pendant longtemps par la violence. Ces quelques remarques ne prétendent nullement traiter « des Siciliennes " en général, mais dresser un tableau de leur situation au moment de la création de l'Association. Car sa naissance se situe dans une période clef, tant par rapport au contexte sociétal que par rapport au " contexte mafieux ». On ne peut en effet ignorer ni l'héritage du rôle des féministes des années 70 ni la période terrible et sanguinaire de la fin des années 70 et du début des années 80 pour Palerme, qui connut alors une recrudescence alarmante de meurtres, notamment avec les premiers assassinats de " servitori dello stato ${ }^{11}$ ", tels que Cesare Terranova, Gaetano Costa, Chinicci ou Dalla Chiesa.

9 C'est dans ce contexte qu'émerge l'Association des femmes siciliennes pour la lutte contre la mafia qui naît officiellement le 27 janvier 1984. Sa formation remonte cependant à la fin des années 70-début des années 80 . Elle résulte en effet d'une initiative de Giovanna Terranova, Caterina Mancuso et Rita Costa qui avaient lancé une pétition populaire adressée au Président de la République, au Président du Conseil et aux gouvernements régionaux de Sicile et de Calabre, demandant à l'État et à toutes les forces politiques de s'occuper réellement du problème mafieux et de faire la lumière sur les délits qui lui sont inhérents. Elles avaient obtenu 30000 signatures en Sicile et en Calabre et créé alors un " Comité des femmes contre la mafia ». Comme le soulignent Norbert Elias et Eric Dunning:

Les chances des hommes d'accéder au pouvoir diminuent et celles des femmes augmentent en proportion chaque fois que les relations dans une société ou une partie de la société deviennent plus pacifiées, lorsque les chances qu'ont les femmes de s'engager ensemble dans l'action rejoignent ou dépassent celles des hommes, et que la ségrégation sexuelle commence à s'effacer ${ }^{12}$.

C'est donc dans ce contexte de violence extrême que des femmes ont décidé de se regrouper pour s'opposer à ce système.

La naissance d'une association féminine

11 La mobilisation initiale des " mères fondatrices » a dépendu de certains facteurs " socioculturels », mais aussi de facteurs biographiques similaires. En effet, Giovanna Terranova, 
Caterina Mancuso et Rita Costa ont en commun la perte de leurs maris, tous les trois « servitori dello stato », et ce dans la même période ; le juge Cesare Terranova est assassiné en septembre 1979, en même temps que Lenin Mancuso, tandis que le juge Gaetano Costa est tué quelques mois après en août 1980. Elles ont un niveau social et scolaire comparables et appartiennent à la même génération, ce qui leur a assuré un niveau élevé d'interconnaissance et un sentiment accru de solidarité. Par la suite, d'autres adhérentes ont rejoint leur association et il s'est formé un " noyau dur » permanent - le conseil de direction et quelques personnalités - et des " satellites » volatiles - les étudiantes par exemple. Leur volonté de tendre à l'élargissement en rassemblant des femmes d'origines diverses est indéniable. Pourtant, malgré leur effort de transversalité, les membres « permanentes " font en majorité partie de la bourgeoisie, voire de la haute bourgeoisie sicilienne. Il faut, en effet, certaines armes pour être en mesure de lutter contre la mafia, à commencer par une "socialisation démocratique ", c'est-à-dire l'intériorisation de discours et de pratiques fondés sur la légalité et les règles de l'État de droit, tel que recourir au détenteur du monopole de la violence légitime - demander justice - et non à celui du monopole de la violence illégitime - vendetta. Ainsi, la seule femme du peuple à faire partie du conseil - qui est composé d'une veuve de juge, d'une avocate, d'une femme médecin, d'une syndicaliste, d'une enseignante, d'une femme politique, etc. - ne peut plus se rendre aux réunions de l'assemblée générale parce qu'elle habite en dehors de Palerme, et que l'essence lui coûte trop cher ; elle est par conséquent exclue de fait, malgré la volonté affichée par les autres - et sincère, me semble-t-il - d'accueillir des femmes de tous milieux. Ceci pose la question dela représentativité. Finalement, même si, durant les procès, l'Association a été présente aux côtés de femmes émanant des milieux mafieux, même si elle a accepté leur adhésion dans les principes après de vifs débats, elle est tendanciellement une lutte d'intellectuelles, un groupement politique qui a, me semble-t-il, constitué un tournant historique concernant l'accession des femmes à la sphère publique sicilienne et qui aujourd'hui survit à ce moment crucial.

\section{LA DYNAMIQUE ASSOCIATIVE FÉMININE}

12 Après avoir esquissé un portrait des femmes qui choisissent de s'investir dans la sphère publique, encore faut-il tenter de comprendre comment et pourquoi fonctionne cette dynamique associative féminine.

L'économie de l'engagement

Dans la société sicilienne, ce passage est lourd de conséquences, surtout au moment de la création de l'association. Les sanctions négatives inhérentes à ce choix sont de nature diverse : d'ordre économique et matériel - perte de l'emploi ou du commerce -, mais aussi d'ordre personnel et intime - rupture avec les amis et la famille. Ce choix correspond donc aussi à un sacrifice intime, la « prise de parole ${ }^{13}$ » étant sanctionnée par l'isolement et par l'exclusion de la vie sociale quotidienne palermitaine. Il représente une rupture profonde, d'autant qu'il nécessite une remise en cause des normes intériorisées depuis l'enfance. Par le coût énorme d'un tel engagement, ces femmes incarnent d'une certaine façon l'idéal-type de la figure du (de la) militant(e), si l'on considère qu'étymologiquement ce terme désigne l'individu qui, tel un soldat, pourrait sacrifier sa vie sur l'autel de sa Cause, d'autant que la décision de dénoncer et de collaborer avec les forces publiques, « [...] est de fait la plus improbable dans l'environnement qui est en général celui des mafias, c'est-à-dire sur fond d'État incapable d'assurer la sécurité de ses citoyens $^{14}$.» 

violence quotidienne ne choisissent pas de s'insérer dans cette lutte. En revanche, il semble qu'il y ait un potentiel féminin de protestation puisqu'à l'inverse des hommes, les Siciliennes ne disposent d'aucun statut dans la mafia et de presque aucun dans l'espace public de l'île. Si en apparence, le coût de la participation à l'action collective est plus important que le bénéfice concret qu'elles peuvent escompter, l'aspect symbolique ne doit pas être négligé. La satisfaction de se conformer à un code moral valorisant peut favoriser l'action publique, la conviction d'œuvrer pour « le bien » ayant des implications sur l'image qu'elles se renvoient à elles-mêmes ; en un sens, «[...] l'activité politique mène bel et bien au développement personnel ${ }^{15}$ ». S'engager dans l'association, c'est aussi entamer un travail de deuil ; deuil du proche, mais aussi deuil de son ancien statut car s'opère une sorte d'évolution du rôle de victime passive et privée à celui d'accusatrice active et publique. D'ailleurs Giovanna Terranova, la présidente, refuse catégoriquement l'expression "veuve de la mafia ", qui la renvoie à une position inactive, et m'explique qu'avant la mort de son mari, elle n'avait aucune vie sociale indépendante : « Avant, j'étais seulement la femme de mon mari ${ }^{16}$ ».

Parallèlement à cette reconstruction identitaire personnelle se dessine une construction identitaire collective, l'émergence d'un " nous ». Les militantes trouvent au sein de l'association une aide psychologique et morale, un lieu à l'intérieur duquel elles peuvent s'exprimer librement, ce qui crée entre elles des phénomènes de solidarité. Elles se reconnaissent alors dans ce " nous, femmes siciliennes ", qui se pose en opposition à un ennemi commun - Cosa Nostra. Car c'est de cette manière que se passe l'intervention, par une conjonction, de logiques personnelles et de logiques communes. Finalement, les adhérentes mobilisent le privé au service du public.

Le vocabulaire de l'engagement

Pour caractériser ce passage au politique, les femmes se réfèrent à un répertoire de justification prévu, presque codifié. Son analyse offre des indications sur la perception que celles-ci ont de leurs propres implications. Le «noyau dur " de ces femmes s'est engagé dans l'Association après l'expérience traumatisante que représente le meurtre d'un proche et justifie par conséquent cette première démarche par le mode affectif. Ainsi expliquent-elles leur décision par le souci de ne pas laisser sombrer dans l'oubli cette mort - l'expression " non dimenticare ", c'est-à-dire "ne pas oublier ", est employée très fréquemment. Cette relation intime à l'engagement associatif s'alimente du sentiment de la dette envers le proche décédé. Elles s'appuient sur un certain nombre de « valeurs » qui se conjuguent avec leurs réactions émotionnelles comme si leur expérience particulière s'entremêlait d'un caractère universaliste, oscillant ainsi, entre devoir de mémoire littérale pour sa propre histoire et devoir de mémoire exemplaire pour l'Histoire ${ }^{17}$. C'est pourquoi, outre leur histoire personnelle, les femmes mobilisent la religion, la morale, ou la « conscience civique ». Michela Buscemi raconte : «Me battre, c'est comme une mission ; maintenant que j'ai fait ce choix, il faut que je continue ma route ${ }^{18}$. ». En effet, bien qu'elles utilisent la mort du proche pour légitimer leur choix, c'est-à-dire l'aspect émotionnel, elles ont également réalisé une forme de rationalisation du discours relatif à leur action ; la rancœur semble par exemple avoir été - délibérément ? - occultée. Ainsi affirment-elles se regrouper autour du projet de la construction d'une société sicilienne sans violence, libérée de la mafia. Dans leurs discours, elles ont donc élaboré une sorte de légitimation de leur action associative par la "morale affective ", mais aussi par la " morale collective civique ». Les femmes se rassemblent autour de cette référence 
constante au bien commun, au devoir envers la société à partir de leur expérience personnelle de la douleur, ce qui accrédite leur action, mais au risque de créer une certaine forme de discours convenu, de rhétorique militante, issue de la construction consensuelle de l'histoire de leur mobilisation. L'impulsion associative semble avoir réalisé un fort degré d'unification symbolique du langage ; il ressort des entretiens comme des interviews accordées aux journalistes - un discours uniforme. Elles légitiment leur action par le biais de la nécessaire intervention citoyenne dans un espace public menacé, renvoyant de cette manière à l'intérêt commun. Afin de faire entendre leur voix, elles ont d'ailleurs instrumentalisé un certain nombre de supports matériels : manifestations et mouvements de protestation, interviews, diffusion d'une sorte de petit fascicule présentant l'Association, son histoire, sa raison d'être et ses actions, etc.

L'instrumentalisation politique du discours

Or, même si ces femmes suscitent spontanément respect et admiration, la recherche consiste à analyser ces prises de position sans complaisance ni malveillance car « il n'y a pas d'indécence à faire des analyses de témoignages et documents quels qu'ils soient ${ }^{19}$. " En l'occurrence, il me semble nécessaire de dépasser ces propos homogènes et de souligner certaines contradictions. Car malgré la volonté clairement exprimée de se constituer en association de femmes, leur discours est sexuellement neutre. En effet, les évocations successives de la morale, du devoir ou de la " conscience civique " ne permettent pas véritablement de clarifier l'introduction du critère sexuel de différenciation ; des hommes auraient pu utiliser les mêmes arguments avec les mêmes termes. Elles revendiquent pourtant fermement leur singularité féminine. Il me semble qu'il y ait à ce niveau, du moins en apparence, une incohérence. Pour la dépasser, Anna Puglisi, qui travaille au Centro siciliano di documentazione Giuseppe Impastato à Palerme, invite à une piste de recherche intéressante lorsqu'elle explique :

Nous avons voulu faire entendre notre voix en tant que femmes, pour trouver un

langage commun et une stratégie commune pour sortir du cercle violence/

résignement/exclusion ${ }^{20}$.

Elle sous-entend que leur lutte, par le biais de l'intervention dans l'espace public, leur permettrait de sortir d'un modèle de société qui leur est largement défavorable, puisqu'il les " exclut " de toute vie publique. Il y a une antinomie apparente dans le fait de demeurer entre femmes tout en aspirant à la participation à la res publica. Cela accrédite en filigrane l'hypothèse selon laquelle elles ne peuvent accéder directement au champ politique, à la citoyenneté selon les mêmes modalités que les hommes. Mais la contradiction s'efface si l'on considère que l'Association des femmes siciliennes pour la lutte contre la mafia a servi de tremplin vers cette vie de la Cité si longtemps inaccessible aux citoyennes. La crise que connaît aujourd'hui l'association semble confirmer cette dernière proposition. Certes, celle-là est en partie due à la baisse générale du taux d'attention - le danger consistant à croire que lorsque la mafia ne tue plus, c'est qu'elle n'est plus -, et à son fonctionnement quasi informel ainsi qu'à son refus de la logique d'entreprise. Mais la chute du nombre d'adhérentes peut également être expliquée par leur évolution dans des associations mixtes ou par leur investissement dans des carrières politiques, et donc par un glissement d'une " petite communauté de citoyennes » vers la « communauté des citoyens ${ }^{21}$ ».

\section{FONCTIONS SOCIALES ET POLITIQUES DE L'ASSOCIATION}

19 Ainsi, l'association serait l'un des facteurs de cette évolution démocratique. Elle aurait constitué un corps intermédiaire entre les citoyennes et l'État. En Sicile, il existe depuis 
bien longtemps un énorme gouffre entre les institutions étatiques et les individu(e)s confronté(e)s quotidiennement aux problèmes économiques et au problème mafieux, surtout pour les femmes qui, comme nous l'avons vu précédemment, étaient écartées de la sphère mafieuse et de la sphère publique sicilienne. Or, même si la plupart d'entre elles adoptent une position ambiguë envers l'État, elles recourent néanmoins à lui par le biais de la justice. D'ailleurs, il semble que l'objet de leurs critiques ne soit pas tant l'État que son dysfonctionnement, ses dérives et ses liaisons dangereuses avec la Mafia. Giovanni Falcone disait même de l'engagement de l'État contre le crime organisé qu'il était :

Émotionnel. Épisodique. Fluctuant. Motivé seulement par l'effet que tel ou tel crime ou, à l'opposé, telle ou telle initiative gouvernementale pourront avoir sur l'opinion. Il s'agit d'une vision myope ${ }^{22}$.

20 Face à cet État discrédité, l'association se présente comme une sorte de relais politique moderne. Souvent les femmes ont avoué que sans l'aide de l'association, elles n'auraient probablement pas eu le courage d'entamer les procédures judiciaires. Elle assure en effet la liaison entre les femmes et les instances étatiques puisqu'elle pose le problème de " l'articulation, de la médiation, de la fonction du politique non institutionnel au politique institutionnel, des espaces publics de proximité aux espaces politiques de délégation, de la société civile à l'État ${ }^{23}$. ». Cette forme de groupement est l'expression d'une liberté inhérente à la démocratie qui permet aux femmes de se familiariser avec les principes de la citoyenneté et de s'exercer à ses pratiques. Par leur engagement, elles évoluent de leur expérience particulière au combat collectif. Ce passage était improbable, étant donné le caractère patriarcal de la société sicilienne, c'est pourquoi il découle initialement des relations interpersonnelles entre les femmes. L'adhésion et les liens dans l'Association se fondent donc sur un registre intimiste. Il en résulte un sentiment d'appartenance à une sorte de microsociété dans laquelle elles peuvent apprendre l'exercice de la « citoyenneté active " par le travail et les relations en groupe et par l'expression politique. La vie associative est une expérience de cohésion sociale, de même que " tout ce qui force l'homme à compter sur autrui, à régler ses mouvements sur autre chose que les impulsions de son égoïsme ${ }^{24}$ ». Cette rencontre suscite entre les femmes des mécanismes de solidarité, à partir desquels émerge un groupe uni par un « destin civique » commun, une identité collective à vocation universelle; c'est la naissance du « nous, citoyennes siciliennes ", marqué par une forte sociabilité interne elle-même fondée sur un idéal partagé et des pratiques communes. Par exemple, le discours fortement unifié est un indice de l'existence d'un véritable groupe. Le « nous » transcende les intérêts particuliers et prétend à l'universalité.

Une fonction de socialisation politique

21 L'Association se révèle être un lieu de socialisation politique, si l'on entend par là le " processus d'inculcation des normes et des valeurs qui organisent les perceptions par les agents sociaux du pouvoir politique (dimension verticale) et du groupe (dimension horizontale) ${ }^{25}$ ». Ce processus concerne particulièrement les membres issus de milieux mafieux, mais aussi aux jeunes et aux enfants vers qui les femmes orientent leur action, notamment en intervenant dans les écoles pour faire naître une "conscience civique nouvelle ", selon leurs termes. Dans De la démocratie en Amérique, Tocqueville avait déjà montré que la forme associative est une force pour la démocratie dans la mesure où les individus peuvent s'y inscrire dans une pratique citoyenne socialisée. Cette forme est d'autant plus pertinente dans une société où l'État de droit n'a jamais pénétré, et dont les femmes sont restées à l'écart. L'Association représente d'ailleurs un tournant important, puisque sa création a été suivie d'une véritable explosion d'associations, de fondations et 
de centres consacrés à l'engagement contre la mafia par un effet " boule de neige »entre autres Laboratorio Zen insieme, le cartel d'associations Palermo Anno Uno, Associazione Nuova resistenza, Associazione palermitana per la pace, Associazione Inventare insieme, etc. L'empressement avec lequel les intéréssé(e) s ont accepté de participer à ma recherche me semble révélateur de l'enjeu politique sous-jacent.

Une étape vers la citoyenneté

L'adhésion à l'Association des femmes siciliennes pour la lutte contre la mafia peut constituer une sorte de passage pour ces femmes, la première marche d'une évolution vers la citoyenneté, redéfinie. Leur participation aux événements politiques s'accroît sensiblement après leur engagement par le biais du vote, de la participation aux manifestations et de l'engagement dans d'autres associations. Par exemple, son « noyau dur » a organisé en collaboration avec d'autres femmes des manifestations pour la paix au Kosovo durant le mois d'avril 1999. Les femmes jusqu'alors exclues représentaient un potentiel de changement. N'étant ni reconnues ni intégrées dans les relations sociales de type clientélaire, elles avaient donc intérêt à remettre en cause, à détruire et à les remplacer par des principes démocratiques. Car l'évocation de la citoyenneté sert souvent de protection aux plus faibles ;

Ceux qui appartiennent à des ethnies ou à des groupes minoritaires, politiquement et juridiquement dominés, ont un intérêt direct à ce que soient reconnus l'égalité juridique et les droits politiques de tous - égalité qui compense, au moins partiellement ou symboliquement, l'infériorité de leur statut. C'est parce que les populations juridiquement et politiquement minoritaires comprennent mieux que les autres ce qui les protège qu'elles ont été particulièrement attachées aux principes de la citoyenneté démocratique ${ }^{26}$.

L'invocation du droit aux principes démocratiques fonctionne alors comme une sorte d'instrument de lutte contre le modèle patriarcal.

Normalement, ces femmes auraient dû soit se taire (omertà), en tant que veuves des " serviteurs de l'État ", soit se venger par le sang (vendetta), en tant que femmes issues de milieux mafieux. Telle est la règle en Sicile. Malgré son caractère oppressif, le système mafieux a pendant longtemps bénéficié d'un certain prestige ou tout au moins d'une acceptation auprès de la population sicilienne. Les normes de la société nationale n'y ont pas été intériorisées comme elles ont pu l'être en Italie du Nord, ce qui explique que les individus aient privilégié leur insertion dans le groupe familial ou clientélaire plutôt que dans une appartenance citoyenne. Dominique Schnapper le souligne : "Dans l'Italie du Sud, pour ne pas aller plus loin, les solidarités familiales n'ont jamais cessé de primer sur les exigences civiques ${ }^{27}$.»

De plus, à Palerme, la massification du chômage entretient l'habitude de " bricolage financier » oscillant entre légalité et illégalité ; la situation chronique des difficultés économiques entretient et aggrave le système clientéliste puisqu'elle nourrit la paupérisation des populations.

L'originalité de ces femmes est donc d'avoir recours à la justice étatique dans une région où la confiance en l'État est extrêmement limitée; elles ne se taisent pas mais dénoncent publiquement les crimes mafieux. Ces femmes passent de la lutte privée au témoignage public, ce qui constitue déjà pour elles une rupture culturelle. Il s'agit d'une remise en cause des règles, des normes mafieuses intériorisées depuis l'enfance, et ce surtout quand il s'agit de femmes issues de milieux mafieux, moins nombreuses à collaborer avec la justice que les veuves d'hommes politiques, magistrats et policiers. Ainsi peut-on se 
demander si on n'assiste pas aujourd'hui avec la multiplication de ces mouvements à une redéfinition de la nature du lien social en Sicile.

\section{BIBLIOGRAPHIE}

Ouvrages sur la mafia italienne

ALAJMo Roberto, Un Lenzuolo contro la mafia, Palermo, Gelka, 1993.

BRICQUET Jean-Louis, « Comprendre la mafia. L'analyse de la mafia dans l'histoire et les sciences sociales », Paris, Politix $n^{\circ} 30$ : Incertitudes italiennes, $2^{\mathrm{e}}$ trimestre 1995.

FALCONE Giovanni, Cosa Nostra, interview recueillie par Marcelle Padovanni, Paris, Édition n ${ }^{\circ} 1 /$ Austral, 1991.

IMPASTATO Felicia Bartolotta, La Mafia in casa mia, Palermo, La Luna, 1987.

LEVI Carlo, Le Parole sono pietre, Torino, Einaudi, 1979.

LONGRIGG Clare, L'Altra metà della mafia, Milano, Ponte alle grazie, 1997.

MADEO Liliana, Donne di mafia. Vittime, complici, e protagoniste, Milano, Tascabili Baldini \& Castoldi, 1994.

MAFAi Simona, Lo CASCio Gigliola, Gugino Carola, OtTAViano Chiara, VitToRelli Beatrice, SALADINo Giuliana, VENUTI Maria, Essere donna in Sicilia, Roma, editori Riuniti, 1976.

Minicucci Maria, « Notes sur la condition féminine dans un village du sud de l'Italie », Femmes de la Méditerranée, Peuples Méditerranéens, n²2-23, janvier-juin 1983.

Mirone Luciano, Le Città della luna. Otto donne sindaco in Sicilia, Soveria Mannelli, Rubbettino, 1997. PINO Marina, Le Signore della droga, Palermo, La Luna, 1988.

PUGLISI Anna, Sole contro la mafia, Palermo, La Luna, 1990.

-, Donne, mafia, e antimafia, Palermo, Csd appunti 7-8, Centro siciliano di documentazione Giuseppe Impastato, 1998.

RizzA Sandra, Una ragazza contro la mafia, Palermo, La Luna, 1993.

SIEBERT Renate, Le donne, la mafia, Milano, Il Saggiatore, 1994.

Ouvrages théoriques

DURKHEIM Émile, Les Règles de la méthode sociologique, Paris, PUF, 1995 (1895).

ELIAS N. et DUNNING E., Sport et civilisation. La violence maîtrisée, Paris, Fayard, 1986.

FORGES J.- F., Éduquer contre Auschwitz, histoire et mémoire, Paris, ESF éditeur, 1997.

Hirschman Albert O, Exit, voice and loyalty (1970), trad. Défection et prise de parole, Paris, Fayard, 1995.

-, Bonheur privé, action publique (1982), Paris, trad. Fayard, 1983.

OLson Mancur, Logique de l'action collective, (1966), trad. rééd. Paris, PUF, 1987. 
SCHNAPPER Dominique, La Communauté des citoyens. Sur l'idée moderne de nation, Paris, Gallimard « NRF essais », 1994.

Todorov Tzvetan, Les Abus de la mémoire, Paris, Seuil, coll. « Arléa Poche », 1998.

\section{NOTES}

1.Extrait du journal de Rita Atria cité dans Sandra Rizza, Una Ragazza contro la mafia - Rita Atria, Palermo, La Luna, 1993, p. 137.

2.Francesca Serio, la mère du syndicaliste Salvatore Carnevale assassiné en 1955 est la première à demander justice pour l'assassinat de son fils.

3.Après la mort de Stefano Leale en 1960 à Palerme, suivi de celle de son fils adoptif en 1962, Serafina Bataglia, compagne du premier et mère du second accuse publiquement les assassins et livre des renseignements très précis sur les activités mafieuses de l'époque.

* Le cas présenté dans cet article a été étudié lors de la préparation du DEA de sociologie de l'École des Hautes Études en Sciences Sociales, Paris, sous la direction de Dominique Schnapper, au cours de l'année universitaire 1998-1999 : « La Transformation de la division sexuelle des rôles sociaux par l'intervention des femmes dans l'espace public. L'association des femmes siciliennes pour la lutte contre la mafia».

4.Giovanni Falcone, Cosa Nostra, interview recueillie par Marcelle Padovani, Paris, Édition n¹/Austral, 1991, p. 89.

5.Entretien de dimanche 18 avril 1999, Balestrate.

6. Notamment: Liliana Madeo, Donne di mafia. Vittime, complici, e protagoniste, Milano, Tascabili Baldini \& Castoldi, 1994 ; Clare Longrigg, L'altra metà della mafia, Milano, Ponte alle Grazie, 1997 ; Marina Pino, Le signore della droga, Palermo, La Luna, 1988, etc.

7.Anna Puglisi, Donne, mafia e antimafia, Palermo, Csd appunti 7-8, Centro siciliano di documentazione Giuseppe Impastato, p. 30.

8.Giovanni Falcone, op. cit., p. 70.

9.Sur ce point, voire Renate Siebert, «E'femmina, però è bella ». Tre generazioni di donne al sud, Torino, Rosenberg \& Sellier, 1991.

10.Marc Dana dans Hérodote. Revue de géographie et de géopolitique, $2^{\mathrm{e}}$ trimestre $1998, \mathrm{n}^{\circ} 89$, Italie. La question nationale, chapitre 10 : «L'indépendantisme sicilien dans le contexte de la crise de l'État italien », p. 182.

11.L'expression « servitori dello stato » — « serviteurs de l'État » - désigne les policiers, carabinieri, hommes politiques, magistrats, etc.

12. Norbert Elias et Eric Dunning, Sport et civilisation. La violence maîtrisée, Paris, Fayard,

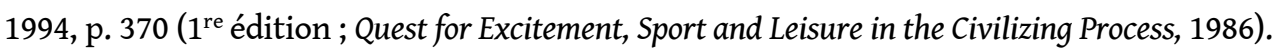
13.J'utilise l'expression « prise de parole » au sens d'Hirschman, « Répond donc à la définition de la prise de parole toute tentative visant à modifier un état de fait jugé insatisfaisant, que ce soit en adressant des pétitions individuelles ou collectives à la direction en place, en faisant appel à une instance supérieure ayant barre sur la direction ou en ayant recours à divers types d'action, notamment ceux qui ont pour but de mobiliser l'opinion publique. ", Albert O. Hirschman, Défection et prise de parole, Paris, Fayard, collection « l'espace du politique », 1995, (1 ${ }^{\text {re }}$ édition en 1970 par Harvard University Press), p. 54.

14.Isabelle Sommier, Les Mafias, Paris, Montchrétien, collection « point clef », 1998, p. 60. 
15.Albert O. Hirschman, Bonheur privé, action publique, Paris, Fayard, collection « l'espace du politique », 1983, p. 157 (édition originale : Shifting Involvements. Private interest and Public Action, Princeton University Press, 1982).

16.Entretien du jeudi 22 avril 1999, Palerme.

17.Voir la distinction établie par Tzvetan Todorov dans Les Abus de la mémoire, Paris, Seuil, collection Arléa Poche, 1998.

18.Reportage réalisé pour Arte par Anne Rougière, reporter à Capa Télévision. 19.Jean-François Forges, Éduquer contre Auschwitz, histoire et mémoire, Paris, ESF éditeur, 1997, p. 39.

20.Anna Puglisi, op. cit., p. 20.

21.Cette dernière expression est empruntée à Dominique Schnapper, La Communauté des citoyens. Sur l'idée moderne de nation, Paris, Gallimard, coll. « NRF essais », 1994.

22.Giovanni Falcone, op. cit., p. 155.

23.L. Maheu, «Identité et enjeux du politique », dans L. Maheu et A. Sales, La Recomposition du politique, Paris, L'Harmattan, Montréal, Presses universitaires de Montréal, 1991, p. 318. 24.Émile Durkheim, De la division du travail social, Paris, PUF, 1991, p. 394.

25.Philippe Braud, Sociologie politique, Paris, LGDJ, 3édition, (« lexique »), 1996, p. 558.

26.Dominique Schnapper, op. cit., p. 147.

27.Dominique Schnapper, op. cit., p. 112.

\section{RÉSUMÉS}

La mafia a toujours fait l'objet de mythes, alimentés notamment par le cinéma et la littérature. Parmi les représentations traditionnellement véhiculées, celle concernant l'idée de la femme sicilienne soumise à son père, à ses frères, puis à son mari est particulièrement vivace. C'est pourquoi l'Association des femmes siciliennes pour la lutte contre la mafia a touché ma curiosité intellectuelle engendrant spontanément un grand nombre d'interrogations relatives à cette spécificité féminine, historiquement déterminante dans la lutte antimafia.

Intervention of women and redefinition of public framework : the case of the Association of Sicilian women for the struggle against the mafia.- The mafia has always been the subject of myths, notably nourrished by cinema and literature. Among the traditional representations, the idea of the submissive Sicilian woman, dominated by her father, her brothers and her husband is particularly lasting. That is why the Association of Sicilian women for the struggle against the mafia cannot but arouse many questions related to this specificity of women, which is historically decisive in the struggle against the mafia.

\section{AUTEUR}

\section{KARINE DELAVIE-BLANCHON}

Karine Delavie-Blanchon est l'auteur d'une maîtrise de science politique à Paris I, puis d'un diplôme d'études approfondies en Sociologie à l'EHESS sous la direction de Dominique Schnapper. 